\title{
Funciones de producción para madera aserrada en la Empresa Forestal Comunitaria en México
}

\section{Sawnwood production functions in the Community Forest Enterprise in Mexico}

\author{
Edgar Arturo Sánchez Moreno1*, José María Salas González, Sergio Pérez Elizalde², \\ Marcos Portillo Vasquez ${ }^{1}$ y José Luis Romo Lozano ${ }^{1}$
}

\begin{abstract}
Three production models for sawnwood were generated in 35 Community Forest Companies (CFEs) located in 10 states of México, which were classified based on variables related to: production inputs, continuous variables, and nominal variables that affect productivity. For Model I the variables $L$ and $K$ were highly significant ( $p$-value $<0.001)$. Variables $L$ and $V D$ in model II were highly significant and variable $K$ was significant at $0.16 \%$ ( $p$-value $<0.05)$. The $C A, C M, C I, P O$ and $P T D$ variables had no effect on productivity. For model III, high significance was obtained for the variables $L$ and $V D(p$-value $<0.001), K$ was significant at $0.95 \%$ ( $p$-value $<0.01)$, Figura Legal Privada (FL2) and Figura Legal Sociedad (FL3) were significant at $0.1 \%$ ( $p$-value $<0.001)$ and $2.9 \%(p-v a l u e$ $<0.05)$, respectively. Competitiveness in the use of resources was measured with the Marginal Product Value (MPV). The hypothesis of non-competitive use for input $L$ in model II $(0.1 \%)$ was rejected. For Model III, Ho $P m g_{L}-P_{L}=0$ was rejected in the CFEs located in the states of Oaxaca and Quintana Roo ( $p$-value <0.05) and Ho: $P \mathrm{Pm}_{K}-P_{K}=0$ was rejected for the CFEs located in Chiapas and Oaxaca ( $p$-value $\left.<0.01\right)$. Finally, the MPV for VD in models II and III using aggregated data at the national level Ho: $P m_{V_{D}}-P_{V D}=0$ was rejected $(p$-value $<0.001)$.
\end{abstract}

Key words: Competitiveness, Cobb-Douglas function, econometric model, productivity, performance to scale, marginal revenue product.

\section{Resumen}

Se generaron tres modelos de producción para madera aserrada en 35 Empresas Forestales Comunitarias (EFC) de 10 estados de México, que se clasificaron a partir de variables relativas (insumos de producción): continuas y nominales que afectan la productividad. Para el modelo I, la mano de obra $(L)$ y el capital $(K)$ fueron altamente significativas ( $p$-valor $<0.001$ ); en el modelo II, lo fueron $L$ y volumen disponible (VD); $K$ resultó significativa al $0.16 \%$ ( $p$-valor < 0.05); Coeficiente de Aserrío anual $(C A)$, Clasificación de madera anual (CM), Capacidad instalada de la industria anualmente $(C I)$, Número de mujeres en puestos operativos al año $(P O)$ y Número de mujeres en puestos de toma de decisiones por año $(P T D)$ no incidieron en la productividad $(p-v a l o r>0.05)$. Para el modelo III $L$ y VD registraron alta significancia; $K$ fue significativa al $0.95 \%(p$-valor $<0.01)$; Figura Legal Privada (FL2) y Figura Legal Sociedad (FL3), lo fueron al $0.1 \%$ ( $p$-valor $<0.001)$ y $2.9 \%(p-v a l o r<0.05)$, respectivamente. La competitividad en el uso de los recursos se determinó con el Valor del Producto Marginal (VPM). Se rechazaron las hipótesis de uso no competitivo para $L$ en el modelo II (0.1 \%); para el modelo III, Ho: $P m g_{L}-P_{L}=0$ en las EFC de Oaxaca y Quintana Roo ( $p$-valor $\left.<0.05\right)$; y Ho: $P m_{K^{-}}-P_{K}=0$ para las de Chiapas y Oaxaca $(p$-valor $<0.01)$. El VPM para $V D$ en los modelos II y III, con datos nacionales, se rechazó Ho: $P m g V D-P V D=0$ con un nivel de significancia de $0.1 \%$.

Palabras clave: Competitividad, función Cobb-Douglas, modelo econométrico, productividad, rendimiento a escala, valor del producto marginal.

Fecha de recepción/Reception date: 27 de noviembre de 2020

Fecha de aceptación/Acceptance date: 27 de julio de 2021

${ }^{1}$ Universidad Autónoma Chapingo. México.

${ }^{2}$ Colegio de Postgraduados. México.

*Autor para correspondencia; correo e: easanchez@colpos.mx 


\section{Introducción}

En México existen 15584 ejidos y comunidades forestales que se denominan núcleos agrarios, y que son poseedores de $45.5 \%$ de la superficie total forestal del país (62 639719 ha) (Reyes et al., 2012; Conafor, 2019a; Frey et al., 2019). Dichas organizaciones crean Empresas Forestales Comunitarias (EFC) para el manejo de los recursos forestales, con el fin de generar beneficios económicos, sociales y ambientales para mejorar su calidad de vida, sin descuidar su viabilidad financiera, competitividad y sustentabilidad ecológica en el largo plazo (Frey et al., 2019); por lo que es fundamental analizar, si las existentes en México cumplen tales propósitos.

Las EFC tienen usos y costumbres que en ocasiones dificultan una administración expedita y no fácilmente acceden a créditos, por lo que dependen de apoyos gubernamentales otorgados por la Comisión Nacional Forestal (Conafor) para aumentar su competitividad y eficiencia; estos se enfocan en disminuir los costos de producción, mejorar la calidad de los procesos, incrementar la eficiencia y agregar valor a sus productos (PNUD, 2017). Frey et al. (2019) evaluaron el efecto de esos apoyos y concluyeron que la certificación forestal impacta positivamente en la productividad $y$, en consecuencia, en el ingreso total de la comunidad.

Estudios recientes sobre ingresos de las EFC muestran resultados diferenciados por una gran variabilidad, atribuible a la diversidad en tamaño, región, productividad y costos (Cubbage et al., 2011; Cubbage et al., 2015a).

En general, las EFC replican un modelo de empresa competitiva para generar empleos y beneficios tanto para sus integrantes, como para las comunidades; mediante la adopción de estrategias de innovación, certificación, de alianzas y mejoras continuas de procesos. Trabajos como los de Cubbage et al. (2013; 2015a; 2015b) y Frey et al. (2019) indican que la competitividad se relaciona con la capacidad de la empresa (de cualquier tipo) de penetrar en el mercado o aumentarlo; lo anterior se estima a través de los costos de producción, ingresos, beneficios y sustentabilidad de cosecha de la 
madera, los cuales explican su rentabilidad y eficiencia. Chandra y Shishodia (2017) y Sasatani (2009) consideran que los indicadores financieros clásicos (rentabilidad del crecimiento, de los activos, del capital y ganancias antes de intereses, impuestos, depreciación y amortización); así como los costos de producción y la rentabilidad son indispensables para medir la competitividad con mayor precisión.

Generalmente, los estudios sobre competitividad empresarial en México no abordan un esquema amplio que involucre aspectos económicos, ambientales y sociales en su conjunto; por ello, el uso de información actualizada (2017 - 2019) de fuentes directas, la especificación de la naturaleza de las inversiones y la forma de organización de las EFC, en la presente investigación constituyen un enfoque novedoso. Sus aportaciones contribuirán a retroalimentar y complementar el análisis de los resultados del Programa para el Fortalecimiento del Manejo Forestal Sustentable con Enfoque de Paisaje (PFMFSP) y de otros estudios como los de Cubbage et al. (2013; 2015a; 2015b) y Frey et al. (2019).

Los indicadores económicos, ambientales y sociales forman parte de la estrategia del PFMFSP para aumentar la competitividad en un mercado local, cuyas importaciones rebasan su propia oferta. De hecho, Cubbage et al. (2015a; 2015b) identifican que el incremento a las importaciones de madera aserrada pone en riesgo la eficiencia y sustentabilidad de las EFC en México.

En el presente estudio se midió el efecto de diversos factores de los ámbitos social (empleos, posición de puestos de trabajo y género) y ambiental (certificación de la cadena de custodia de productos forestales y del buen manejo forestal) sobre la producción y productividad de las EFC, mediante la teoría de la empresa y del desarrollo forestal sustentable. El primer objetivo de esta investigación consistió en determinar funciones de producción tipo Cobb-Douglas para medir el efecto de las variables que afectan la productividad. El segundo fue medir la competitividad a partir del Valor del Producto Marginal y determinar los rendimientos a escala para los insumos mano de obra, capital y volumen disponible. 


\section{Materiales y Métodos}

\section{Materiales}

La información provino del PFMFSP de la Conafor para los años de 2017 a 2019, correspondiente a 35 EFC ubicadas en 10 estados de México: Chiapas, Chihuahua, Durango, Estado de México, Jalisco, Michoacán, Oaxaca, Puebla, Quintana Roo y Veracruz; además de, los subsidios que se les asignaron en los mismos años (Conafor, 2017; 2018; 2019b). Las mediciones y monitoreo de indicadores del PFMFSP se agruparon en tres criterios, lo que sirvió para identificar mejoras en la competitividad, acceso a mercado e igualdad de género de acuerdo al Programa de las Naciones Unidas para el Desarrollo (PNUD) (PNUD, 2017).

Los subsidios correspondieron al Programa Nacional Forestal (Pronafor) mediante apoyos directos a la inversión del comercio y la industria forestal, a la administración, producción y comercialización e integración y organización de redes de valor forestal, así como subsidios indirectos para gastos operativos, certificaciones nacionales e internacionales y mercadotecnia (Conafor, 2017; 2018; 2019b).

\section{Métodos}

\section{Funciones de producción}

Se utilizó la función de producción $(Q)$ de tipo Cobb-Douglas con dos factores de la producción: capital $(K)$ y mano de obra $(L)$ dada por el modelo:

$$
Q=e^{\beta_{0}} L^{\beta_{1}} K^{\beta_{2}}
$$


Donde:

$\beta_{i}=$ Parámetros de la función

$\beta_{1}=$ Elasticidad de $L$

$\beta_{2}=$ Elasticidad de $K$

$K=$ Producto de la capacidad instalada anual (en pies tabla turno-1) por el desgaste de capital, referido a la depreciación de la maquinaria (20\%), obtenido a partir del costo de producción unitario $\left(\$ \mathrm{pt}^{-1}\right)$ en el proceso de aserrío

$L=$ Número de empleos directos por turno de 8 horas por 240 días laborales al año

El modelo (1) puede linealizarse al aplicar el logaritmo natural $(I n)$, con lo que se obtiene:

$$
\ln Q=\beta_{0}+\beta_{1} \ln L+\beta_{2} \ln K
$$

Dado un conjunto de observaciones de las variables $Q, K$ y $L$, en $n_{i}$ EFC ubicadas en el estado $i$, los parámetros de la ecuación (2) se estiman con el modelo de regresión con efecto mixto dado por:

$$
\ln Q_{i j}=u_{i}+\beta_{0}+\beta_{1} \ln L_{i j}+\beta_{2} \ln K_{i j}+\epsilon_{i j}, i=1, \ldots, p, j=1, \ldots, n_{i}
$$

Donde:

$\epsilon_{i j}=$ Errores normales, independientes e idénticamente distribuidos con media 0 y varianza $\sigma^{2}$

$u_{i}=$ Efecto aleatorio normal del Estado $i$-ésimo, con media 0 y varianza $\sigma_{u}^{2}$; este término modela implícitamente la correlación entre observaciones, $\rho=\sigma_{u}^{2} /\left(\sigma^{2}+\sigma_{u}^{2}\right)$, inducida por la cercanía geográfica, factores ambientales comunes y condiciones socioeconómicas similares 
El ajuste del modelo mixto (3) se realizó con el paquete Ime4 del programa estadístico R (R Core Team, 2020). Para verificar la normalidad se utilizó la prueba de ShapiroWilk y la homogeneidad de varianza de los errores con las pruebas de homocedasticidad de Barttlet y Levine.

El modelo base (1) se extendió para incluir otras variables continuas y categóricas que inciden en el nivel de producción. En general, la función de producción propuesto, de acuerdo con Frey et al. (2019) fue:

$$
c Q=e^{\beta_{0}} \Pi_{i=1}^{m} X_{i}^{\beta_{i}} \Pi_{j=1}^{n} Y_{j}^{\gamma_{j}} \Pi_{k=1}^{r} e^{\delta_{k} Z_{k}}
$$

Donde:

$Q=$ Cantidad producida en millares de pie tabla (Mpt $=1000 \mathrm{pt}$ ) por año

$\beta_{0}, \beta_{i}, Y_{j}, \delta_{k}=$ Parámetros de la función

$X_{i}=$ Cantidades de insumos de producción

$Y_{j}=$ Variables continuas que afectan la productividad

$Z_{k}=$ Variables nominales $\mathrm{u}$ ordinales que afectan la productividad

El modelo (4) es una variación empírica de la forma funcional tipo Cobb-Douglas (Frey et al., 2019) que se transforma en el modelo lineal de la forma:

$$
\ln Q=\beta_{0}+\sum_{i=1}^{m} \beta_{i} \ln X_{i}+\sum_{j=1}^{n} \gamma_{j} \ln Y_{j}+\sum_{k=1}^{r} \delta_{k} Z_{k}
$$

Con base en la expresión (5) se definen tres modelos etiquetados en orden de complejidad. Con el modelo (6) es posible determinar el efecto de los factores de producción $K$ y $L$; en el modelo (7) se agregan los factores relacionados con el nivel tecnológico, la disponibilidad de madera y la inclusión de mujeres en el proceso 
productivo. Con el modelo (8) se evalúa el efecto de los subsidios, la certificación de madera y la figura legal con la que está constituida la EFC. De este modo, al prescindir de los subíndices para simplificar la notación, la estructura de los modelos de regresión por estimar como extensiones del modelo original (Ec. 1) se presenta a continuación:

$$
\begin{aligned}
\ln Q= & u+\beta_{0}+\beta_{1} \ln L+\beta_{2} \ln K+\varepsilon \\
\ln Q= & u+\beta_{0}+\beta_{1} \ln L+\beta_{2} \ln K+\beta_{3} \ln V D+\gamma_{1} \ln C A+\gamma_{2} \ln C M+ \\
& \gamma_{3} \ln C I+\gamma_{4} \ln P O+\gamma_{5} \ln P T D+\varepsilon \\
\ln Q= & u+\beta_{0}+\beta_{1} \ln L+\beta_{2} \ln K+\beta_{3} \ln V D+\gamma_{1} \ln C A+\gamma_{2} \ln C M+\gamma_{3} \ln C I+\gamma_{4} \ln P O+\gamma_{5} \ln P T D+ \\
& \delta_{1} S+\delta_{2} C E R T+\delta_{3} F L+\varepsilon
\end{aligned}
$$

Donde:

$Q=$ Producción anual (Mpt)

$L=$ Trabajo (empleos totales al año)

$K=$ Capital anual (pesos)

$V D=$ Volumen de madera disponible anual ( $\mathrm{m}^{3}$ rollo)

$C A=$ Coeficiente de aserrío anual (\%)

$C M=$ Clasificación de madera anual (\%)

$C I=$ Capacidad instalada de la industria anualmente (pt turno ${ }^{-1}$ )

$P O=$ Número de mujeres al año en puestos operativos

$P T D=$ Número de mujeres al año en puestos de toma de decisiones

$S=$ Concepto de subsidios de Pronafor (número de apoyos año-1)

CERT $=$ Certificación $\operatorname{CoC}(0,1)$

$F L=$ Figura legal $(F L 1=$ Ejido o Comunidad, $F L 2=$ Privada y $F L 3=$ Sociedad $)$ 
Para cuantificar la producción de unidades físicas en el proceso de aserrío, se realizó el modelado de la función de producción a partir de los modelos $(6,7$ y 8$)$.

\section{Valor del Producto Marginal}

En la teoría microeconómica, una empresa es competitiva si el valor del producto marginal (VPM) de cada insumo es igual a su precio. El VPM es el ingreso adicional que obtiene una empresa por contratar una unidad de trabajo adicional $(L)$ o una unidad de capital $(K)$. Es decir, si las EFC buscan la máxima competitividad, los ingresos generados por una unidad adicional de insumo deberían ser iguales a su costo; esto es, el VPM del insumo, menos el precio será igual a cero (Salvatore, 1983; Mas et al., 1995; Varian, 2006). A partir de la ecuación (4), la hipótesis de competitividad en términos del valor del producto marginal está dado por:

$$
\begin{gathered}
P_{Q}\left(\frac{\partial Q(L, K)}{\partial X_{f}}\right)-P_{f}=0 \\
P_{Q}\left[e^{\beta_{0}} \Pi_{i=1}^{m} X_{i}^{\beta_{i}} \Pi_{j=1}^{n} Y_{j}^{\gamma_{j}} \Pi_{k=1}^{r} e^{\delta_{k} Z_{k}}\right]-P_{f}=0
\end{gathered}
$$

Donde:

$P_{Q}=$ Precio de mercado de la madera aserrada ponderado al precio nacional $\left(\$ \mathrm{pt}^{-1}\right)$ $P_{f}=$ Precio de insumo ( $\$$ salario $^{-1}, \$$ capital $^{-1}$ ) obtenido a partir del costo de producción (30 \% del costo de producción para salario y $20 \%$ del costo de producción para capital)

$\beta_{0}, \beta_{f}, Y_{j}, \delta_{k}=$ Parámetros de la función

A partir de la ecuación (4), también es posible construir la hipótesis de competitividad de la EFC en función del VPM para la mano de obra $(L)$ : 


$$
\begin{gathered}
P_{Q}\left(\frac{\partial Q(L, K)}{\partial L}\right)-P_{L}=0 \\
P_{Q}\left[\alpha_{1} Q(L, K)\left(u+\alpha_{0}+\alpha_{1} \ln L+\alpha_{2} \ln K\right)^{\frac{1}{\lambda}-1} L^{-1}\right]-P_{L}=0
\end{gathered}
$$

De forma análoga, la hipótesis de competitividad para la EFC en función del VPM para el capital $(K)$ es:

$$
\begin{gathered}
P_{Q}\left(\frac{\partial Q(L, K)}{\partial L}\right)-P_{L}=0 \\
P_{Q}\left[\alpha_{2} Q(L, K)\left(u+\alpha_{0}+\alpha_{1} \ln L+\alpha_{2} \ln K\right)^{\frac{1}{\lambda}-1} K^{-1}\right]-P_{K}=0
\end{gathered}
$$

Donde:

$p_{Q}\left(\frac{\partial Q(L, K)}{\partial L}\right)=$ Valor marginal del producto

$P L=$ Precio de mano de obra

$P K=$ Precio de capital

$P V D=$ Precio del volumen unitario de madera

Con las expresiones (11) y (12) se realizó la prueba de las hipótesis para VPM de los insumos $L$ y $K$ y $V D$ para determinar la competitividad nacional y estatal, respectivamente. Para contrastar las hipótesis se utilizó la prueba de Wald para 
restricciones no lineales (Greene, 2011) mediante el programa estadístico R (R Core Team, 2020).

Adicionalmente, se probaron las hipótesis de Ho: $\beta_{k}+\beta_{L}=1$ para determinar rendimientos constantes a escala para los insumos $K$ y $L$ (Salvatore, 1983; Gould y Lazear, 1998).

\section{Resultados}

\section{Funciones de producción}

Las expresiones de las funciones de producción finales derivadas de los modelos 6, 7 y 8 , dadas por los modelos I, II y III se listan a continuación:

$$
\begin{aligned}
\ln Q=- & 10.371+0.690 \ln L+0.795 \ln K \\
\ln Q=- & 6.382+0.475 \ln L+0.320 \ln K+0.481 \ln V D \\
\ln Q=- & 5.724+0.442 \ln L+0.252 \ln K+0.520 \ln V D+0.267 \ln F L 1+ \\
& 0.781 \ln F L 2+0.369 \ln F L 3
\end{aligned}
$$

Los valores de los parámetros por variable para las funciones de producción, el nivel de significancia, el resultado de las pruebas estadísticas y de las hipótesis se muestran en el Cuadro 1 ; entre paréntesis se indica el $p$-valor. 
Cuadro 1. Funciones de producción Cobb-Douglas para madera aserrada.

\begin{tabular}{|c|c|c|c|}
\hline \multirow{2}{*}{ Variable/prueba } & \multicolumn{3}{|c|}{ Modelo } \\
\hline & $\mathbf{I}$ & II & III \\
\hline $\ln L$ & $0.690(0.0) * * *$ & $0.475(0.0) * * *$ & $0.442(0.0) * * *$ \\
\hline $\ln K$ & $0.795(0.0) * * *$ & $0.320(0.0016) * *$ & $0.252(0.0095)^{* *}$ \\
\hline $\ln V D$ & & $0.481(0.0) * * *$ & $0.520(0.0)^{* * *}$ \\
\hline FL1 & & & $0.267(0.0801)$ \\
\hline FL2 & & & $0.781(0.001)^{* * *}$ \\
\hline FL3 & & & $0.369(0.029) *$ \\
\hline Constante & $-10.371(0.0)^{* * *}$ & $-6.382(0.0) * * *$ & $-5.724(0.0) * * *$ \\
\hline \multicolumn{4}{|l|}{ Estadísticos } \\
\hline$R^{2}$ & 0.816 & 0.848 & 0.845 \\
\hline$F_{0^{a}}$ & $0.988(0.503)$ & $0.990(0.635)$ & $0.984(0.248)$ \\
\hline Bartlett/Levine ${ }^{b}$ & $14.685(0.1)$ & $1.475(0.168)$ & $1.323(0.235)$ \\
\hline \multicolumn{4}{|l|}{ Prueba estadística } \\
\hline$F_{1}^{c}$ & $23.86(0.0)^{* * *}$ & $2.93(0.0865)$ & $8.56(0.005)^{* *}$ \\
\hline$I M g_{L}-P_{L}^{d}$ & $2117.497(0.392)$ & $642.42(0.012)^{*}$ & $297.183(0.138)$ \\
\hline$I M g_{K}-P_{K}^{d}$ & $5.962(0.471)$ & $0.752(0.181)$ & $0.752(0.960)$ \\
\hline$I M g_{V D}-P_{V D}{ }^{d}$ & & $-708.236(0.0)^{* * *}$ & $-828.57(0.0) * * *$ \\
\hline
\end{tabular}

${ }^{a} F_{o}$ Prueba de normalidad Shapiro-Wilk, no se rechaza la normalidad ( $p$-valor $\left.>0.001\right)$;

${ }^{b}$ Prueba de homocedasticidad de varianzas que indicó que la varianza de los residuos es constante ( $p$-valor $>0.001$ ), para los modelos II y III no se rechaza la hipótesis de homocedasticidad; 'Prueba de hipótesis Ho: $\beta_{L}+\beta_{K}=1$; dPrueba de hipótesis de Ho: $P m g_{L}-P=0$ para datos agregados a nivel nacional; $* p<0.05$;

$$
* * p<0.01 ; * * * p<0.001 \text {. }
$$

Los estadísticos de la prueba de hipótesis para el VPM a nivel estado para las variables mano de obra $(L)$ y capital $(K)$ del modelo I, se presentan en el Cuadro 2, con Ho: $P m g_{L}-P_{L}=0$ y Ho: $P m g_{K}-P_{K}=0$, respectivamente. 
Cuadro 2. Estadísticos para la prueba de VPM con el Modelo I.

\begin{tabular}{lcc}
\hline \multicolumn{1}{c}{ Estado } & Estimación $\boldsymbol{V P} \boldsymbol{M}_{\boldsymbol{L}}(\boldsymbol{p}$-val) & Estimación $\boldsymbol{V P M _ { \boldsymbol { K } } ( \boldsymbol { p } \text { -val) }}$ \\
\hline Chiapas & $1115.438(0.358)$ & $3.710(0.497)$ \\
Chihuahua & $1034.867(0.362)$ & $6.386(0.443)$ \\
Durango & 4031.0750 .397 & $7.680(0.471)$ \\
Edo. México & $3147.397(0.397)$ & $11.999(0.430)$ \\
Jalisco & $2219.260(0.372)$ & $4.462(0.479)$ \\
Michoacán & $1943.497(0.390)$ & $1.440(0.637)$ \\
Oaxaca & $1624.518(0.424)$ & $6.181(0.469)$ \\
Puebla & $2365.610(0.385)$ & $7.020(0.466)$ \\
Quintana Roo & $3153.318(0.373)$ & $8.878(0.449)$ \\
Veracruz & $3092.774(0.390)$ &
\end{tabular}

En el Cuadro 3 se presentan los estadísticos de prueba de hipótesis para el VPM a nivel estado para las variables $L, K$ y $V D$ del Modelo II, con $\mathrm{Ho}: P m g_{L}-P_{L}=0$, Ho: $P m g_{K}-P_{K}=0$ y Ho: $P m g_{V D}-P_{V D}=0$, respectivamente.

Cuadro 3. Estadísticos para la prueba de VPM con el Modelo II.

\begin{tabular}{|c|c|c|c|}
\hline Estado & $\begin{array}{c}\text { Estimación } \\
\text { VPML (p-val) }\end{array}$ & $\begin{array}{c}\text { Estimación } \\
V P M_{K}(p-v a l)\end{array}$ & $\begin{array}{c}\text { Estimación } \\
\text { VPMVD }(p-v a l)\end{array}$ \\
\hline Chiapas & $195.653(0.040)^{*}$ & $-0.1744(0.491)$ & $-346.505(0.0009) * * *$ \\
\hline Chihuahua & $320.119(0.053)$ & $0.884(0.132)$ & $-408.536(0.0005) * * *$ \\
\hline Durango & $1178.74(0.006)^{* *}$ & $1.0442(0.150)$ & $-716.938(0) * * *$ \\
\hline Edo. México & $377.987(0.195)$ & $1.2909(0.075)$ & $-46.939(0.799)$ \\
\hline Jalisco & $356.280(0.045)^{*}$ & $0.0578(0.876)$ & $-53.295(0.723)$ \\
\hline Michoacán & $507.136(0.014) *$ & $0.1563(0.698)$ & $-648.341(0) * * *$ \\
\hline Oaxaca & $461.268(0.001)^{* * *}$ & $-0.4049(0.056)$ & $-776.435(0) * * *$ \\
\hline Puebla & $609.375(0.005) * *$ & $0.6137(0.205)$ & $-787.506(0) * * *$ \\
\hline Quintana Roo & $927.197(0.001)^{* * *}$ & $0.8459(0.146)$ & $-4007.601(0)^{* * *}$ \\
\hline Veracruz & $784.274(0.009) * *$ & $1.2457(0.064)$ & $-791.833(0) * * *$ \\
\hline
\end{tabular}


En el Cuadro 4 se indican los estadísticos de prueba de hipótesis para el VPM a nivel estado para las variables $L, K$ y $V D$ del Modelo III, con $\mathrm{Ho}: P m g_{L}-P_{L}=0$; Ho: $P m g_{K}-P_{K}=0$; Ho: $P m g_{V D}-P_{V D}=0$, respectivamente. A diferencia del Modelo II, en este modelo se probó el efecto de la variable figura legal a nivel estado y no fue significativa estadísticamente.

Cuadro 4. Estadísticos para la prueba de VPM (Modelo III).

\begin{tabular}{|c|c|c|c|}
\hline Estado & $\begin{array}{l}\text { Estimación } \\
\operatorname{VPM}(p-v a l)\end{array}$ & $\begin{array}{c}\text { Estimación } \\
V P M_{K}(p-v a l)\end{array}$ & $\begin{array}{c}\text { Estimación } \\
V P M_{V D}(p-v a l)\end{array}$ \\
\hline Chiapas & $63.298(0.383)$ & $-0.538(0.002)^{* *}$ & $-537.153(0) * * *$ \\
\hline Chihuahua & $147.292(0.227)$ & $0.091(0.827)$ & $-557.230(0) * * *$ \\
\hline Durango & $698.691(0.057)$ & $0.293(0.595)$ & $-771.934(0) * * *$ \\
\hline Edo. México & $-208.213(0.213)$ & $-0.017(0.963)$ & $-591.969(0) * * *$ \\
\hline Jalisco & $91.627(0.471)$ & $-0.4343(0.069)$ & $-402.964(0.004)^{* *}$ \\
\hline Michoacán & $267.964(0.116)$ & $-0.2887(0.334)$ & $-760.214(0) * * *$ \\
\hline Oaxaca & $378.242(0.016) *$ & $-0.548(0.004)^{* *}$ & $-758.686(0) * * *$ \\
\hline Puebla & $283.657(0.122)$ & $-0.044(0.901)$ & $-896.420(0) * * *$ \\
\hline Q. Roo & $525.086(0.033) *$ & $0.122(0.777)$ & $-4253.510(0)^{* * *}$ \\
\hline Veracruz & $248.478(0.272)$ & $0.201(0.653)$ & $-945.969(0) * * *$ \\
\hline
\end{tabular}

\section{Discusión}

En el Modelo I las variables $L$ y $K$ en el grupo de factores de producción fueron altamente significativas ( $p$-valor $<0.001$ ). Además, en el Modelo II otras variables de este tipo que determinaron la productividad en las EFC fueron $V D$ y $L$; en tanto que, la variable $K$ fue significativa a $0.16 \%$ ( $p$-valor < 0.05$)$. CA, CM, CI, PO y PTD no tuvieron efecto alguno en la productividad ( $p$-valor $>0.05$ ); lo que se explicaría porque 
no tienen relación directa con la productividad, pues más bien se refieren al resultado de estrategias de mercado, de mejoras tecnológicas y de la cuota de género.

Los coeficientes en una función del tipo Cobb-Douglas son las elasticidades de los insumos $L$ y $K$ en los modelos I, II y III (Cuadro 1 ), y representan la variación porcentual del nivel de producto $(Q)$ frente a variaciones porcentuales en el trabajo y el capital. Las elasticidades de los insumos $L$ y $K$ para producir un nivel de producto $Q$ (Modelo I, Cuadro 1) muestran mayor significancia estadística que cuando se agregan al modelo otras variables como $V D$, las cuales afectan la producción y la productividad (Modelo II, Cuadro 1). Esto es relevante, si se decide utilizar modelos con más variables que expliquen el efecto en la producción de los insumos, como posibles mejoras productivas en el uso de los factores. La significancia estadística de la variable $K$ disminuye al agregar otras como VD y $F L$ (modelos II y III, Cuadro 1 ).

Para el Modelo III, los factores relativos a la figura legal (propiedad privada y sociedad) constituyen el incremento porcentual en la producción de madera aserrada de una EFC, de manera que para empresa privada: $\left(\mathrm{e}^{0.781}-1\right) 100=118 \%$, en tanto que para la EFC con figura legal sociedad: $\left(e^{0.369}-1\right) 100=44 \%$. Lo anterior es relevante, puesto que los ejidos y comunidades forestales son más de $85 \%$ del régimen de propiedad forestal, con respecto a la pequeña propiedad y sociedad. Las variables $F L 1, S$, y $C$ no tuvieron efecto en la productividad de las EFC ( $p$-valor > $0.05)$. En análisis futuros es recomendable que se usen valores monetarios para $S$ en lugar del número de subsidios.

En relación al análisis del $V P M$ para $L$ y $K$ con los datos agregados a nivel nacional, se rechazó la hipótesis Ho: $P m g_{L}-P_{L}=0$ para el Modelo II (Cuadro 1), con un nivel de significancia de $0.1 \%$ ( $p$-valor < 0.05). También, se determinó que no existe significancia estadística para las hipótesis $\mathrm{Ho}: P m_{L}-P_{L}=0$ y $\mathrm{Ho}: P m g_{K}-P_{K}=0$ ( $p$ valor > 0.05) en el análisis del VPM a nivel estado (Modelo I, Cuadro 2). Para el Modelo I (Cuadro 3), al determinar la competitividad de las EFC se rechazó Ho: $\mathrm{Pmg}_{L^{-}}$ $P_{L}=0$ en las EFC de Chiapas, Jalisco y Michoacán ( $p$-valor $\left.<0.05\right)$; Durango, Puebla y Veracruz ( $p$-valor $<0.1$ ); Oaxaca y Quintana Roo ( $p$-valor $<0.001)$. Un valor 
estimado mayor a cero (Cuadro 3), se interpreta como unas EFC no competitivas y que subutilizan el insumo $L$; lo que significa que el $V P M$ de $L$ es mayor al precio de mercado del recurso $L$. Para el Modelo III, se rechaza Ho: $P m g_{L}-P_{L}=0$ en las EFC ubicadas en los estados de Oaxaca y Quintana Roo ( $p$-valor<0.05). De forma similar, se rechaza Ho: $P m g_{K}-P_{K}=0$ para las EFC de Chiapas y Oaxaca $(p$-valor $<0.01)$.

Respecto al análisis de VPM para VD en los modelos II y III con datos agregados a nivel nacional, se rechaza Ho: $P m_{V D}-P_{V D}=0$ con un nivel de significancia de $0.1 \%$ ( $p$-valor < 0.001). El Modelo II rechaza Ho: $P m_{V D}-P_{V D}=0$ para todos los estados ( $p$-valor $<0.001$ ), excepto el Estado de México y Jalisco en los que no se obtuvo significancia estadística.

Las EFC localizadas en Chiapas, Chihuahua, Durango, Michoacán, Oaxaca, Puebla, Quintana Roo y Veracruz, cuando se obtiene un valor estimado menor a cero (Cuadro $3)$, se interpreta que sobreutilizan el insumo $V D$, lo cual significa que el $V P M$ de $V D$ es menor al precio de mercado del recurso VD. Para el Modelo III se rechaza Ho: $P m g_{V D}-P_{V D}=0$ para las EFC de Chiapas, Chihuahua, Durango, Estado de México, Jalisco, Michoacán, Oaxaca, Puebla, Quintana Roo y Veracruz ( $p$-valor < 0.001); por lo que tales EFC son no competitivas en el uso del recurso VD (Cuadro 4).

La relación del $V P M$ y del costo del factor es útil para determinar el uso competitivo de los recursos; sin embargo, no se registró significancia estadística que indique la existencia de uso no competitivo de los insumos $L$ y $K$ a nivel nacional para los modelos I y III, ni para $K$ del Modelo II. Esto sugiere que no hay evidencia sobre la sub o sobreutilización de ambos insumos en el proceso productivo, lo cual es consistente con la maximización del ingreso de la empresa. Resultado que contrasta con el obtenido por Frey et al. (2019), quienes señalan que las EFC son competitivas en el uso de recursos y en el rendimiento a escala.

Los estadísticos de varianza de los datos agregados y a nivel estado indican que son no significativos. No obstante, al comparar los datos agregados y por estado para el Modelo II, los estados de Chiapas, Jalisco y Michoacán mantienen la significancia en el uso de $L$ a $5 \%$ ( $p$-valor < 0.5); mientras que Durango, Puebla y Veracruz aumentan el nivel de significancia al $1 \%$ ( $p$-valor $<0.1)$; Oaxaca y Quintana Roo al $0.1 \%$ ( $p$ - 
valor < 0.001). El Estado de México y Jalisco no alcanzan significancia estadística en el análisis VPM para VD ( $p$-valor $>0.05)$. Los otros estados mantienen la significancia al $0.1 \%$ ( $p$-valor $<0.001)$, al igual que para los datos agregados. En el Modelo III solo Oaxaca y Quintana Roo registran significancia al $5 \%$ ( $p$-valor < 0.05 ) en el análisis VPM para $L$. Contrariamente a los resultados estadísticos de datos agregados, Chiapas y Oaxaca obtienen una significancia en el análisis VPM para $K$ de $1 \%$ ( $p$-valor $<0.01)$, y todos los estados presentan significancia de $0.1 \%$ ( $p$-valor $<0.001$ ) en el análisis VPM para VD.

Esto puede deberse a que la gran variabilidad en los registros de las variables surge al agrupar las EFC por estado. Por ejemplo, en Chihuahua el valor de $Q$ anual fue: máxima $=4709.88$, mínima $=94.89$ y media $=2392 \mathrm{Mpt} \mathrm{año-1;}^{-1}$ cifras sustancialmente mayores a la producción de Puebla, cuyo $Q$ anual fue: máximo $=1332.02$, mínimo $=402.70$ y media $=771.051 \mathrm{Mpt}$ año-1 . El valor de las 35 EFC para $Q$ fue: máximo $=6$ 952.380, mínimo $=90.956$ y media $=1$ 613.229 Mpt año-1.

La prueba de hipótesis Ho: $\beta_{k}+\beta_{L}=1$ para determinar rendimientos no constantes a escala para los insumos $L$ y $K$ y los resultados de las elasticidades de los factores de producción $L$ y $K$ reflejan que ningún modelo tiene rendimientos constantes a escala (Estadístico $F_{1}$ en Cuadro 1 ). De hecho, para el Modelo I se obtuvo un rendimiento creciente a escala $\left(\beta_{L}+\beta_{K}>1\right)$; sin embargo, disminuye al agregar variables continuas ( VD, CA,CM,CI, PO y PTD) y nominales ( $S, C E R T$ y $F L$ ) que afectan la productividad en los modelos II y III, respectivamente; por ello, los rendimientos son decrecientes a escala para $\left(\beta_{L}+\beta_{K}<1\right)$, lo que significa que, en promedio, las EFC estan operando bajo rendimientos decrecientes a escala en el corto plazo; tendencia similar a la descrita por Niquidet y Nelson (2010).

En este estudio, se tuvo alta variabilidad de la información de las EFC analizadas, semejante a lo citado por Cubbage et al. (2015b); las únicas variables con poca variabilidad entre empresas fueron $C A(\mu=0.518, \sigma=0.051)$ y $C M(\mu=0.062$, $\sigma=0.083$ ). Es posible que dicha variabilidad entre empresas asociadas a $C I, C O, P O$, $P T D, C E R T$ influyó para que no incidieran en la productividad. Por ejemplo, la variable 
$C A$ en el Modelo II, no tuvo efecto en la producción; la única variable continua con impacto en la productividad fue $V D$, lo cual es cercano a lo obtenido por Frey et al. (2019) para la variable inventario total de madera de pino y oyamel por aprovechar en las EFC en México.

Es importante destacar que el apoyo gubernamental mediante subsidios y la certificación forestal no tuvo efecto en la producción, lo que coincide con los resultados de Frey et al. (2019) para volumen cosechado. Aunque la información del presente estudio tuvo alta variabilidad, la bondad de ajuste fue buena para los modelos, ya que fluctuó entre 81.60 y $84.60 \%$.

\section{Conclusiones}

El modelo tipo Cobb-Douglas como una expresión particular de la función de producción es útil para construir modelos económicos que representan la producción, como la de madera aserrada en unidades físicas. Los modelos económicos I, II y III desarrollados en este estudio pueden contribuir a definir políticas para fomentar la industria maderera por conducto de los factores con mayor impacto, tales como Trabajo, Capital y Volumen disponible de madera.

No existe uso competitivo en los recursos $L, K$ y $V D$ para las 35 EFC analizadas y ubicadas en 10 estados de la república mexicana.

Las variables $V D$ y $F L(F L 1, F L 2$ y $F L 3)$ tienen efecto en la productividad para los modelos II y III, respectivamente. Las EFC conformadas como empresas privadas y sociedades aportan $118 \%$ y $44 \%$ de la producción, respectivamente. La figura legal de ejido aporta solo 30 \% en la producción.

Las funciones de producción generadas son homogéneas de grado $n>1$ con rendimientos crecientes a escala para el Modelo I y homogéneas de grado $n<1$ con rendimientos decrecientes a escala para los modelos II y III. 
Las variables continuas $C A, C M, C I, P O$ y $P T D$ y nominales $(S, C E R T)$ no tienen ningún efecto en la productividad. Aunque el coeficiente de asierre y la capacidad instalada se relacionan con el nivel tecnológico de la industria, no contribuyen en el incremento de la productividad. La variable clasificación de madera es una estrategia para incrementar el valor de la madera con escasos efectos prácticos. Los puestos operativos para mujeres y en la toma de decisiones que son relativos a la cuota de género, tampoco afectan la productividad; sin embargo, en proyectos de inversión apoyados por el Banco Mundial y Conafor se privilegian aquellos que cumplen con la certificación del buen manejo forestal y la cuota de género.

No fue posible identificar el efecto de los subsidios integrados en un solo concepto en la producción, porque se analizó un periodo de tiempo corto ( 3 años) y los impactos de los apoyos directos e indirectos tardan más tiempo visualizarse.

\section{Agradecimientos}

El primer autor agradece al Conacyt por la beca otorgada para realizar los estudios de doctorado. Al Ing. Mario A. Mosqueda Vázquez de la Coordinación de Producción y Productividad en la Comisión Nacional Forestal (Conafor) quien proporcionó parte de la información para realizar esta investigación.

\section{Conflicto de intereses}

Los autores declaran no tener conflictos de intereses.

\section{Contribución por autor}

Edgar Arturo Sánchez Moreno: diseño de la investigación, colecta y descripción de información, procesamiento de la información, escritura del documento; José María Salas González: apoyo en la descripción de información y análisis de resultados; 
Sergio Pérez Elizalde: aporte de la metodología y modelos estadísticos, análisis de resultados; Marcos Portillo Vásquez: apoyo en la selección de funciones de producción, de costos e ingresos; José Luis Romo Lozano: apoyo en el análisis de información sobre precios de productos e insumos y redacción del documento.

\section{Referencias}

Comisión Nacional Forestal (Conafor). 2017. Resultados de la Convocatoria del Programa Apoyos para el Desarrollo Forestal Sustentable para el año 2017. México. https://www.conafor.gob.mx/apoyos/index.php/inicio/app_apoyos\#/detalle/2017/6 8 (4 de noviembre de 2020).

Comisión Nacional Forestal (Conafor). 2018. Resultados de la Convocatoria del Programa Apoyos para el Desarrollo Forestal Sustentable. México. https://www.conafor.gob.mx/apoyos/index.php/inicio/app_apoyos\#/detalle/2018/7 3 (4 de noviembre de 2020).

Comisión Nacional Forestal (Conafor). 2019a. Estado que guarda el sector forestal en México. SEMARNAT. Guadalajara, Jal., México. 422 p.

https://www.gob.mx/conafor/es/documentos/estado-que-guarda-el-sector-forestalen-mexico-2019?idiom=es (4 de noviembre de 2020).

Comisión Nacional Forestal (Conafor). 2019b. Resultados de la Convocatoria del Programa Apoyos para el Desarrollo Forestal Sustentable. https://www.conafor.gob.mx/apoyos/index.php/inicio/app_apoyos\#/detalle/2019/79 (4 de noviembre de 2020). 
Cubbage, F. W., R. R. Davis y G. Frey. 2011. Guía para la evaluación Económica y Financiera de Proyectos Forestales Comunitarios en México. Banco Mundial Región de Latinoamérica y el Caribe. Documento de Trabajo Forestal Latinoamericano. Núm. 2.

https://www.researchgate.net/publication/311964643 (2 de diciembre 2020).

Cubbage, F., R. Davis, D. Rodríguez P., G. Frey, R. Mollenhauer, Y. Kraus., I. A. González H., H. Albarrán H., A. M. Salazar C. y D. N. Chemor S. 2013. Competitividad y acceso a mercados de empresas forestales comunitarias en México. Program on Forests (PROFOR). Washington, DC., USA. 132 p.

Cubbage, F., R. Davis. D. Rodríguez P., R. Mollenhauer, Y. Kraus E., G. Frey. I. A. González H., H. H. Albarrán H., M. Salazar C. and D. N. Chemor S. 2015a. Community Forestry Enterprises in Mexico: Sustainability and Competitiveness. Journal of Sustainable Forestry 34(6-7):623650. Doi:10.1080/10549811.2015.1040514.

Cubbage, F., R. Davis, D. Rodríguez P., Y. Kraus E., R. Mollenhauer and G. Frey. 2015b. Timber production cost and profit functions for community forests in Mexico. Tropical Forestry Handbook. The World Bank for Reconstruction and Development/The World Bank. Doi:10.1007/978-3-642-41554-8_222-2.

Chandra, B. S. and M. Shishodia. 2017. Agribusiness competitiveness Applying analytics, Typology and measurements to Africa. Discussion paper. 01648. International Food Policy Research Institute (IFPRI). Washington, D.C. USA. 35 p.

Frey, G., F. Cubbage, T. P. Holmes, G. Reyes-Retana, R. R. Davis, C. Megevand, D. RodríguezParedes, Y. Kraus-Elsin, B. Hernández-Toro and D. N. Chemor-Salas. 2019. Competitiveness, certification, and support of timber harvest by community forest enterprises in Mexico. Forest Policy and Economics 107 (10): 11. Doi:10.1016/j.forpol.2019.05.009.

Gould, J. P. y E. P. Lazear. 1998. Teoría Microeconómica. Fondo de Cultura Económica. México, D. F., México. 870 p. 
Greene, W. H. 2011. Econometric Analysis. 7th edition. Prentice Hall Upper Saddle River, NJ, USA. 1188 p.

Niquidet, K. and H. Nelson. 2010. Sawmill production in the interior of British Columbia: a stochastic ray frontier approach. Joumal of Forest Economics 16(4):257-267. Doi: 10.1016/j.jfe.2010.04.001.

Mas C., A., M. D. Whinston and J. R. Green. 1995. Microeconomic Theory. Oxfor University Press. New York, NY, USA. 985 p.

Programa de las Naciones Unidas para el Desarrollo (PNUD). 2017. Documento del Proyecto "Fortalecimiento del Manejo Forestal Sustentable con Enfoque de Paisaje". CDMX, México. 135 p. https://info.undp.org/docs/pdc/Documents/MEX/PRODOC.pdf (20 de noviembre de 2020).

R Core Team. 2020. R: A language and environment for statistical computing. R Foundation for Statistical Computing, Vienna, Austria. https://www.R-project.org/._(24 de octubre de 2020).

Reyes J., A., P. Gómez J., O. Muis R., R. Zavala, A. Ríos G. y O. Villalobos. 2012. Atlas de propiedad social y servicios ambientales en México. Instituto Interamericano de Cooperación para la Agricultura. Cooperación Técnica Registro Agrario Nacional - Instituto Interamericano de Cooperación para la Agricultura. México, D. F., México. 157 p.

Salvatore, D. 1983. Microeconomía. Teoría y 475 problemas resueltos. Serie Schaum. McGraw-Hill. México, D. F., México. 344 p.

Sasatani, D., 2009. APFSOS II: National Competitiveness Index of the Forest Products Industry in the APR. FAO. Bangkok, Thailand. $114 \mathrm{p}$.

Varian H., R. 2006. Microeconomía Intermedia. Un enfoque actual. Ed. Antoni Bosch. Barcelona, España. 789 p.

\section{(c) (7) (8)}

Todos los textos publicados por la Revista Mexicana de Ciencias Forestales -sin excepción- se distribuyen amparados bajo la licencia Creative Commons 4.0 Atribución-No Comercial (CC BY-NC 4.0 Internacional), que permite a terceros utilizar lo publicado siempre que mencionen la autoría del trabajo y a la primera publicación en esta revista. 